\title{
Dietary grape polyphenol resveratrol increases mammary tumor growth and metastasis in immunocompromised mice
}

\author{
Linette Castillo-Pichardo ${ }^{1,2}$, Luis A Cubano ${ }^{3}$ and Suranganie Dharmawardhane ${ }^{1 *}$
}

\begin{abstract}
Background: Resveratrol, a polyphenol from grapes and red wine has many health beneficial effects, including protection against cardiovascular and neurodegenerative diseases and cancer. However, our group and others have provided evidence for a dual cancer promoting or inhibitory role for resveratrol in breast cancer, dependent on estrogenic or antiestrogenic activities. Moreover, much of the inhibitory effects of resveratrol have been reported from studies with high non-physiological concentrations.
\end{abstract}

Methods: We investigated the effects of a range of concentrations $(0.5,5,50 \mathrm{mg} / \mathrm{kg}$ body weight) of resveratrol on mammary tumor development post-initiation, using immunocompromised mice.

Results: Our findings suggest promotion of mammary tumor growth and metastasis by resveratrol at all concentrations tested in tumors derived from the low metastatic estrogen receptor (ER)a(-), ERß(+) MDA-MB-231 and the highly metastatic ER(-) MDA-MB-435 cancer cell lines. Additionally, the activity of the migration/invasion regulator Rac, which we have previously shown to be regulated by resveratrol in vitro, was measured in tumors from resveratrol treated mice. Our results show a significant induction of tumoral Rac activity and a trend in increased expression of the Rac downstream effector PAK1 and other tumor promoting molecules following resveratrol treatment.

Conclusion: Taken together, our findings implicate low concentrations of resveratrol in potential promotion of breast cancer. Therefore, this study illuminates the importance of further delineating resveratrol's concentration dependent effects, particularly in breast cancer, before it can be tested in the clinic or used as a dietary supplement for breast cancer patients.

Keywords: Breast cancer, Resveratrol, Metastasis, Grape polyphenols, Rac

\section{Background}

Dietary consumption of grapes has for long been associated with a decrease in the incidence of cardiovascular diseases and cancer. The beneficial properties of grapes and its products are attributed to polyphenolic compounds that have been shown to have cardioprotective, neuroprotective, anticancer, anti-inflammation, anti-ageing, and antimicrobial properties $[1,2]$. Among the grape and red wine polyphenols, resveratrol (3,4',5-trihydroxy-trans-stilbene) has received a great deal of attention due to a plethora of

\footnotetext{
* Correspondence: su.d@upr.edu

'Department of Biochemistry, School of Medicine, University of Puerto Rico, Medical Sciences Campus, PO Box 365067, San Juan, Puerto Rico Full list of author information is available at the end of the article
}

in vitro and in vivo studies on its cancer chemopreventive and therapeutic properties. Resveratrol has been shown to affect initiation, promotion, and progression of tumorigenesis by modulating cell division and growth, apoptosis, angiogenesis, and metastasis [3-5].

Among the mediators of resveratrol's anticancer effects, the most widely studied are the mitogen activated protein kinase (MAPK), phosphatidyl inositide 3-kinase (PI3-K)/ Akt, and Nuclear Factor kappa B (NFkB) pathways $[4,5]$. Resveratrol is particularly important for breast cancer since it has been shown to exert both estrogenic or antiestrogenic effects and binds to estrogen receptors ER $\alpha$ and ER $\beta$ with comparable affinity, but with 7,000-fold lower affinity than estrogen [6-8]. Resveratrol-mediated

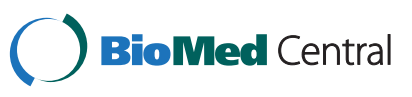


regulation of extracellular regulated kinase (ERK)1/2, c-Jun N-terminal kinase (JNK), and p38 MAPKs has been shown to promote apoptosis of breast cancer cells [9-11]. Resveratrol inhibited cell proliferation and ER $\alpha$ mRNA and protein levels in MCF-7 breast cancer cells through a mechanism dependent on p38 MAPK and p53 regulation [12]. In addition to inhibition of cancer initiation and progression, resveratrol has also been implicated in reduction of metastasis. Resveratrol has been shown to inhibit cell migration/invasion and metastasis in several types of cancer, including breast cancer $[13,14]$. Moreover, resveratrol is able to chemosensitize several types of cancers to chemotherapy. Its potential as a chemosensitization agent is due to the regulation of many signaling molecules including drug transporters, cell survival and cell proliferation regulators, and members of the $\mathrm{NFKB}_{\mathrm{B}}$ and signal transducer and activator of transcription (STAT)3 signaling pathways [15].

However, most studies on the anti-cancer activities of resveratrol have focused on high, non-physiologically relevant, concentrations; whereas the effects of low concentrations, achieved through the diet, on cancer progression have been poorly studied. Our group has shown that depending on the concentration used, resveratrol can act as an estrogenic or an anti-estrogenic compound. We have reported an inhibitory role for high concentrations of resveratrol and a promotional role for estrogen and low concentrations of resveratrol in breast cancer cell migration, extension of actin structures that promote cell migration, and the activity of Rac, a key signaling protein that promotes the actin cytoskeleton rearrangements driving cell migration $[16,17]$.

Despite its many beneficial effects, resveratrol's bioavailability is low as a result of its rapid metabolism in mammals [3]. However, due to its therapeutic potential, the use of novel delivery systems to increase resveratrol's bioavailability is being explored [18,19]. In mouse models of cancer, resveratrol has shown variable results depending on the concentration, where early reports showed that resveratrol prevented mammary tumorigenesis and decreased mammary growth of MDA-MB-231 mammary tumors $[6,20]$. However, recent studies have shown either no effect of treatment with resveratrol in vivo $[21,22]$ or pro-cancer effects in a MDA-MB- 435 model, where resveratrol abrogated the effects of paclitaxel treatment [23].

A number of clinical trials are ongoing on the potential of resveratrol as an anti-cancer compound [24,25]. However, there is a pressing need to further understand the effects of resveratrol in pre-clinical studies, especially with established cancers, given that cancer patients often use resveratrol as a dietary supplement. Because of its low bioavailability, and since resveratrol's dual estrogenic/antiestrogenic role seems to be concentration dependent, it is also important to delineate its anticancer effects at a broad range of concentrations.

Herein we have investigated the effects of resveratrol in the regulation of mammary tumor growth and metastasis at a range of concentrations in a mouse model of breast cancer, using the low metastatic $\operatorname{ER} \alpha(-), \operatorname{ER} \beta(+)$ MDA-MB-231 and the highly metastatic ER(-) MDAMB-435 cancer cell lines. The concentrations selected for this study range from dietary to pharmacological, where it is possible to achieve $0.5-5 \mathrm{mg} / \mathrm{kg}$ BW resveratrol concentrations via dietary consumption. In our study, in mice with an average bodyweight of $20 \mathrm{~g}, 5 \mathrm{mg} / \mathrm{kg} \mathrm{BW}$ of resveratrol in a $100-\mu \mathrm{L}$ gavage volume equates to $\sim 4.38 \mu \mathrm{M}$ resveratrol. These concentrations may be found in dietary components rich in grape polyphenols such as red wine [14]. Interestingly, our findings implicate low concentrations of resveratrol in promotion of mammary tumor growth and metastasis, and identify Rac and other cancerpromoting molecules as potential regulators of resveratrolmediated effects in mammary tumors.

\section{Methods \\ Animals}

Hairless (severe combined immunodeficiency) SCID or athymic nu/nu (or nude) female mice, 5 to $6 \mathrm{wk}$ old (Charles River Laboratories, Inc., Wilmington, MA) were maintained under pathogen-free conditions in Hepa-filtered cages under controlled light (12 h light and dark cycle), temperature $\left(22-24^{\circ} \mathrm{C}\right)$, and humidity (25\%). Throughout the experiment, the animals were provided with sterile AIN 76-A phytoestrogen-free diet (Tek Global, Harlan Teklad, Madison, WI) and water ad libitum. This project was approved by the Institutional Animal Care and Use Committee, Universidad Central del Caribe.

\section{Tumor establishment}

Green fluorescent protein (GFP) tagged-MDA-MB-231 $(\mathrm{ER} \alpha(-), \operatorname{ER} \beta(+))$ or a metastatic variant of GFPMDA-MB-435 (ER $(-))$ cells $\left(\sim 1 \times 10^{6}\right)$ in Matrigel (BD Biosciences, San Jose, CA) were injected into the fourth right mammary fat pad (only one site per mouse) under isofluorane inhalation to produce orthotopic primary tumors as described in [26]. After tumor establishment (1 wk post-inoculation), animals were randomly divided into experimental treatment groups $(n=10-12$ per treatment group). The GFP-MDA-MB-231 cells were inoculated into SCID mice, while the GFP-MDA-MB-435 cells were inoculated into nude mice. Both cell lines were a kind gift of Dr. Danny Welch, The University of Alabama at Birmingham, AL. SCID mice were used for the study conducted with MDA-MB-231 cells to ensure uniform tumor take. For the MDA-MB-435 study, nude mice were used as we previously obtained uniform tumor 
take with this model [26]. Moreover, the aggressiveness and high metastatic potential of the MDA-MB-435 cell line would have caused increased tumor burden in the SCID strain. All injected sites developed into palpable tumors and none regressed below the original signal.

The origin of MDA-MB- 435 cell line has been questioned by studies that show expression of melanomaassociated genes [27]. However, MDA-MB-435 cells are derived from a breast cancer patient, express breast differentiation-specific proteins, and secrete milk lipids [28]. Therefore, the simplest conclusion is that MDA-MB435 is a very poorly differentiated breast carcinoma. A recent publication supports this idea by providing evidence that suggests the MDA-MB- 435 cell line is indeed a poorly differentiated, aggressive breast cancer cell line that expresses both epithelial and melanocytic markers [29].

\section{Diet administration}

Hairless SCID or athymic nu/nu female mice were orally gavaged either with vehicle (90\% neobee oil, 10\% ethanol), or $0.5,5$, or $50 \mathrm{mg} / \mathrm{kg}$ body weight (BW) resveratrol in a $100 \mu \mathrm{L}$ volume every day (5 days/wk). Treatments continued until sacrifice at day 108 for the study performed on GFP-MDA-MB-231 breast cancer cells and at day 44 for the study performed on GFP-MDA-MB-435 breast cancer cells. The study performed with GFP-MDA-MB- 435 cells had to be terminated earlier due to high tumor burden caused by resveratrol in this highly metastatic and aggressive breast cancer cell line.

\section{Whole body fluorescence image analysis}

Mammary tumor growth was quantified as changes in integrated density of GFP fluorescence, using methods developed by Hoffman and co-workers [30-32] and previously described by us in [26,33-35]. Mice (under anesthesia) were imaged one week following breast cancer cell inoculation (on the first day of diet administration) and once a week thereafter. A 300 Watt power source with two optical delivery systems fitted with excitation filters (470/ $40 \mathrm{~nm}$ ) was used for whole body imaging of GFP fluorescence (LT99D2, Lightools Research, Encinitas, CA). Images were captured with a Spot II charge-coupled device (CCD) camera (Diagnostic Instruments, Sterling Heights, MI) mounted with a 530/25 nm emission filter (Chroma Technology, Rockingham, VT).

Tumor fluorescence intensities were analyzed using Image J software (National Institutes of Health, Bethesda, MD). The integrated density of each tumor was computed using threshold values set to detect the fluorescence from the primary tumor area and not the metastatic lesions. Relative tumor growth was calculated as the integrated density of fluorescence of each tumor on each imaging day relative to the integrated density of fluorescence of the same tumor on the first day of diet administration.

\section{Analysis of metastases}

Following sacrifice, lungs, kidneys, livers, and bones (femurs) were excised and immediately stored in liquid $\mathrm{N}_{2}$. Stored organs were thawed and analyzed using an Olympus MV10 fluorescence macro zoom system microscope and images acquired with an Olympus DP71 digital camera as described in $[26,34,35]$. Each organ was imaged on both sides. The fluorescent lesions (green component of RGB images) were quantified for integrated density of fluorescent pixels using Image J software.

\section{Western blot analysis of tumors}

Flash frozen MDA-MB-231-derived primary tumors were lysed using a homogenizer (Brinkmann Polytron, Mississauga, ONT, Canada) as previously reported by us [35]; and total protein quantified using the Precision Red protein assay kit (Cytoskeleton, Inc. Denver, CO). Equal total protein amounts were resolved on SDS-PAGE gels and western blotted using anti-Akt, anti-JNK, antiPAK1, or anti-GFP (Cell Signaling Technology, Inc., Danvers, MA) antibodies. The integrated density of positive bands was quantified using Image $J$ software and normalized with GFP to ensure quantification of tumor proteins.

\section{Western blot analysis of cells}

Quiescent MDA-MB-231 or MDA-MB-435 cells were treated with vehicle, or $0.1,0.5$, or $5 \mu \mathrm{M}$ resveratrol for $15 \mathrm{~min}$. Cells were immediately lysed and total protein was quantified using the Precision Red protein assay kit (Cytoskeleton, Inc., Denver, CO). Equal total protein amounts were western blotted using

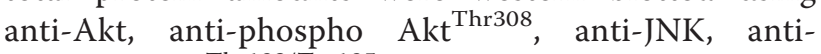

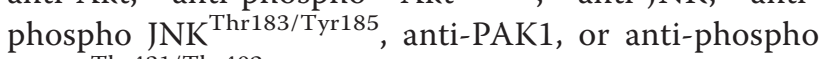

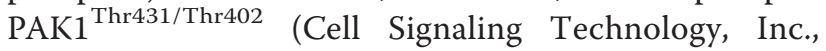
Danvers, MA) antibodies. The integrated density of positive bands was quantified using Image J software.

\section{Rac activity assays}

Flash frozen mammary tumors were lysed using a homogenizer (Brinkmann Polytron, Mississauga, ONT, Canada) as previously described by us [35] and total protein was quantified using the Precision Red protein assay kit (Cytoskeleton, Inc. Denver, CO). Active Rac was pulled down using beads coupled to GST-p21-activated kinase (PAK)-Cdc42/Rac interactive binding (CRIB) motif (GSTPAK-PBD beads) (Cytoskeleton, Denver, $\mathrm{CO}$ ) as described in [36], and detected from western blots immunostained with an anti-Rac antibody (Cell Signaling Technology, Inc., Danvers, MA). Positive bands were imaged using a VersaDoc system (Bio-Rad, Hercules, CA) and quantified using Image J software. Rac activity was determined as the Rac-GTP bound to the PAK-CRIB domain as a function of total Rac in a cell lysate. 


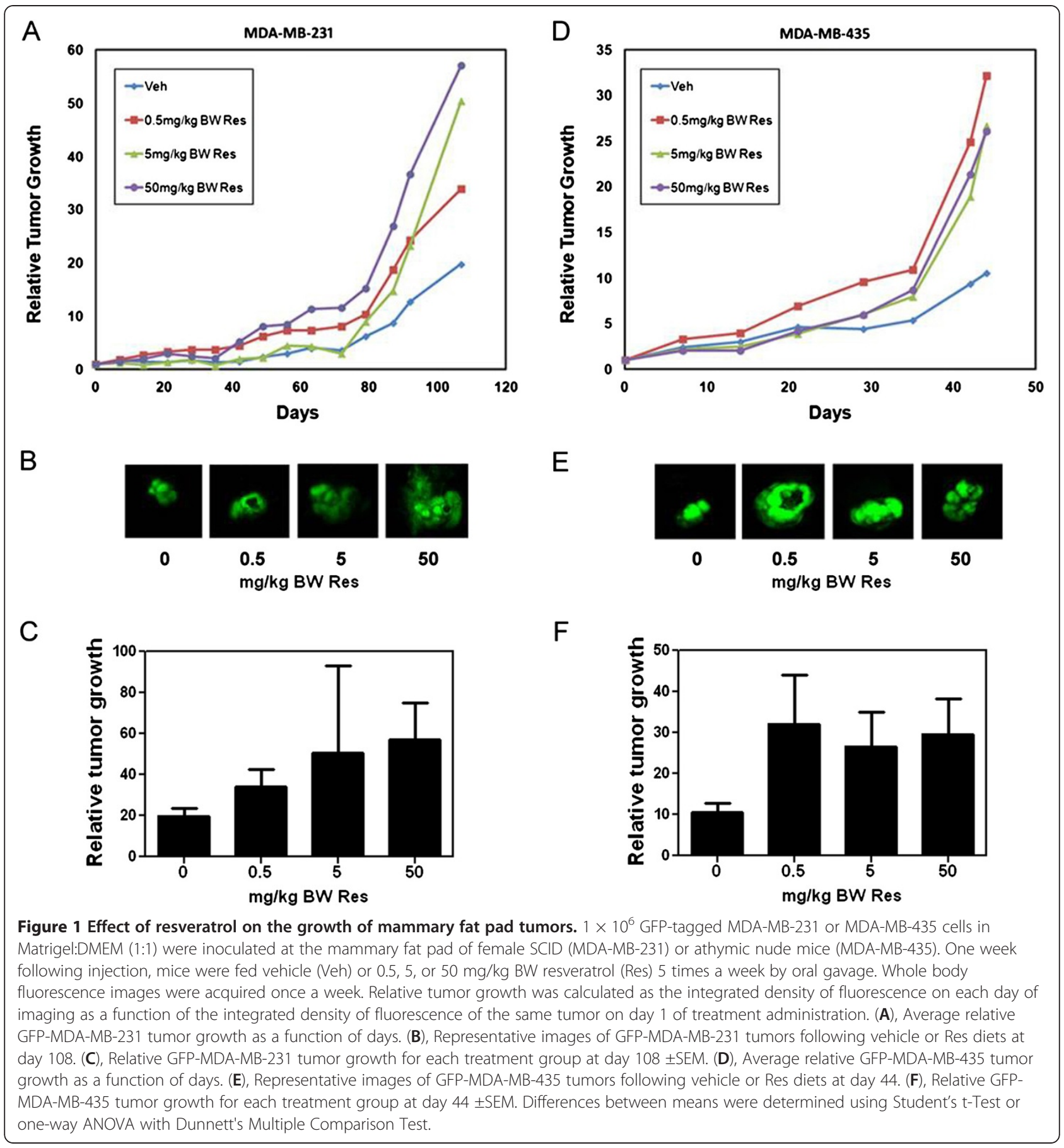

\section{Statistical analysis}

Data are expressed as the mean \pm SEM. Statistical analyses were done using Microsoft Excel and GraphPad Prism $^{\circledR}$. Differences between means were determined using Student's t-Test or one-way ANOVA with Dunnett's Multiple Comparison Test; and considered to be statistically significant at $p \leq 0.05$.

\section{Results and discussion}

Our group has previously reported the dual effects of resveratrol in ER(-) breast cancer cell migration and invasion, while others have shown that resveratrol functions as an anti-estrogen in ER(+) mammary cancer cell lines and rodent models [6]. Our studies with $\operatorname{ER} \alpha(-), E R \beta(+)$ MDA-MB-231 breast cancer cell line demonstrated that 

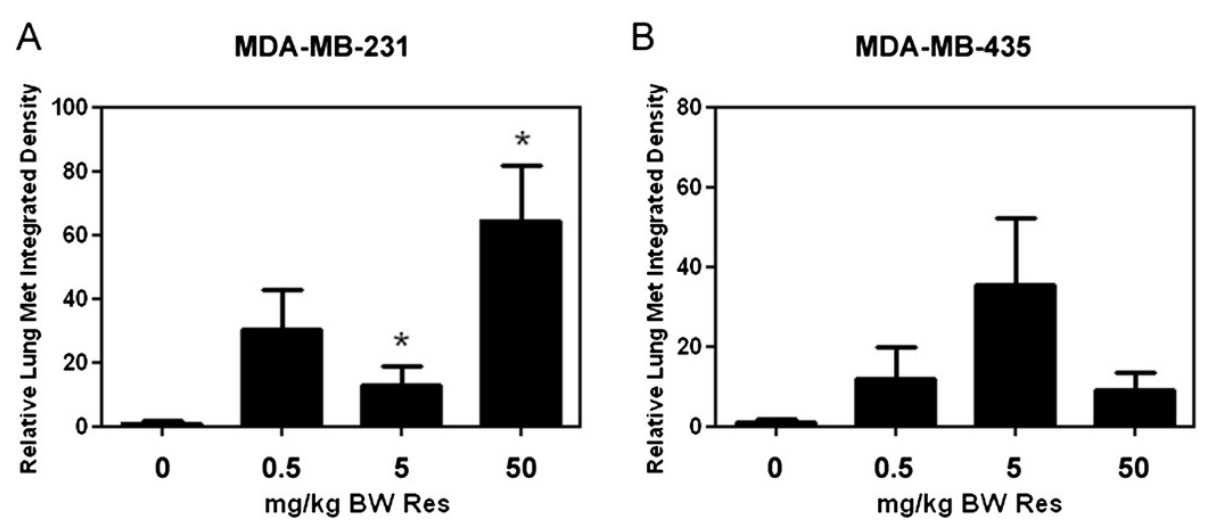

Figure 2 Effect of resveratrol on lung metastasis. Following necropsy, lungs were excised from mice with GFP-MDA-MB-231 or GFP-MDA-MB-435 mammary tumors that received vehicle or $0.5,5$, or $50 \mathrm{mg} / \mathrm{kg}$ BW Res diets and analyzed for metastases by fluorescent microscopy followed by quantitative image analysis. (A) and (B), Relative integrated density ( \pm SEM) of fluorescent metastatic foci for vehicle or Res treated mice from the study performed with (A), MDA-MB-231 and (B), MDA-MB-435 cells (2-9 mice/group). Differences between means were determined using Student's t-Test. Asterisk denotes statistical significance at $p \leq 0.05$.

resveratrol at $50 \mu \mathrm{M}$ acts in an anti-estrogenic manner to reduce cell migration while, resveratrol at $5 \mu \mathrm{M}$ acts similar to estrogen inducing cell migration, invasion, and formation of lamellipodia [36]. Lamellipodia are actin structures found at the leading edge of migrating cells that are under Rac regulation [37]. We reported that $50 \mu \mathrm{M}$ resveratrol decreases Rac activity, whereas estrogen and $5 \mu \mathrm{M}$ resveratrol increases Rac activity in breast cancer cells. Moreover, using breast cancer cells expressing dominant-negative Rac, we demonstrated that signaling to the actin cytoskeleton by low concentrations of resveratrol was under Rac regulation [36]. Since, breast cancers often lose ER expression during progression, we tested the effects of resveratrol in both $\operatorname{ER} \beta(+)$ and $\operatorname{ER}(-)$ metastatic breast cancer cells MDA-MB-231 and MDAMB-435 in vivo.

Many in vivo studies supporting the anti-cancer effects of resveratrol have used high, non-physiologically relevant, concentrations of resveratrol of $50-800 \mathrm{mg} / \mathrm{kg} \mathrm{BW}$ [6,38-40]. However, the effects of low concentrations of resveratrol, which are critical for a better understanding of the possible effect of dietary resveratrol in cancer and for dosing recommendations, have been poorly addressed at the in vivo scenario. Therefore low, physiologically relevant, concentrations of resveratrol $(0.5$ and $5 \mathrm{mg} / \mathrm{kg} \mathrm{BW})$ and a moderate concentration $(50 \mathrm{mg} / \mathrm{kg} \mathrm{BW})$ were used to test resveratrol's effect on the progression of breast cancer.

The bioavailability of resveratrol is considered to be low as per data on plasma levels of resveratrol following dietary consumption. Studies have shown that administration of $20 \mathrm{mg} / \mathrm{kg}$ resveratrol to rats, mice, or rabbits demonstrated a very short half-life (10 minutes) and reached circulating levels of less than $1 \mu \mathrm{M}$ very rapidly [20]. Mice administered $100 \mathrm{mg} / \mathrm{kg}$ of resveratrol achieved a maximum serum concentration of $2277 \mathrm{ng} /$ $\mathrm{mL}$ at $0.25 \mathrm{~h}$ and $\sim 42 \mathrm{ng} / \mathrm{mL}$ at $24 \mathrm{~h}$ [18]. However, others have shown that, administration of as little as $50 \mu \mathrm{g} / \mathrm{kg}$ body weight of resveratrol per day for 3 months to rats achieved serum levels as high as $8.0 \mu \mathrm{M}$ [39]. In the present study, we administered low to moderate $(0.5$, 5 , and $50 \mathrm{mg} / \mathrm{kg} \mathrm{BW)} \mathrm{concentrations} \mathrm{of} \mathrm{resveratrol} \mathrm{daily}$ $5 \times$ a week for 1.5 and 4 months in the MDA-MB-435 and MDA-MB231 studies, respectively. Therefore, we expect the resveratrol levels in the plasma to be low but consistent, and higher in the mammary tissue, as per previous studies that have shown resveratrol levels in plasma are low due to high absorption by tissues [41].

In SCID mice with MDA-MB-231 mammary fat pad tumors, oral gavage of resveratrol resulted in a nonsignificant, but evident increase in tumor growth of $\sim 2$-fold at $0.5 \mathrm{mg} / \mathrm{kg} \mathrm{BW}$ resveratrol and $\sim 2.5$-fold at

Table 1 Effects of resveratrol on metastasis of GFP-MDA-MB-435 mammary tumors

\begin{tabular}{|c|c|c|c|c|c|c|c|c|}
\hline & \multicolumn{4}{|c|}{ Percentage of mice with metastases } & \multicolumn{4}{|c|}{ Average integrated density of fluorescent metastatic foci } \\
\hline & \multirow[t]{2}{*}{ Vehicle } & \multicolumn{3}{|c|}{ Resveratrol (mg/kg BW) } & \multirow[t]{2}{*}{ Vehicle } & \multicolumn{3}{|c|}{ Resveratrol (mg/kg BW) } \\
\hline & & 0.5 & 5 & 50 & & 0.5 & 5 & 50 \\
\hline Kidneys & $0 \%(0 / 7)$ & $14 \%(1 / 7)$ & $10 \%(1 / 10)$ & $22 \%(2 / 9)$ & 0 & 0.21 & 0.12 & 0.29 \\
\hline Liver & $29 \%(2 / 7)$ & $29 \%(2 / 7)$ & $10 \%(1 / 10)$ & $22 \%(2 / 9)$ & 0.24 & 2.4 & 0.70 & 0.20 \\
\hline Bone & $0 \%(0 / 7)$ & $0 \%(0 / 7)$ & $20 \%(2 / 10)$ & $22 \%(2 / 9)$ & 0 & 0 & 0.038 & 0.02 \\
\hline
\end{tabular}



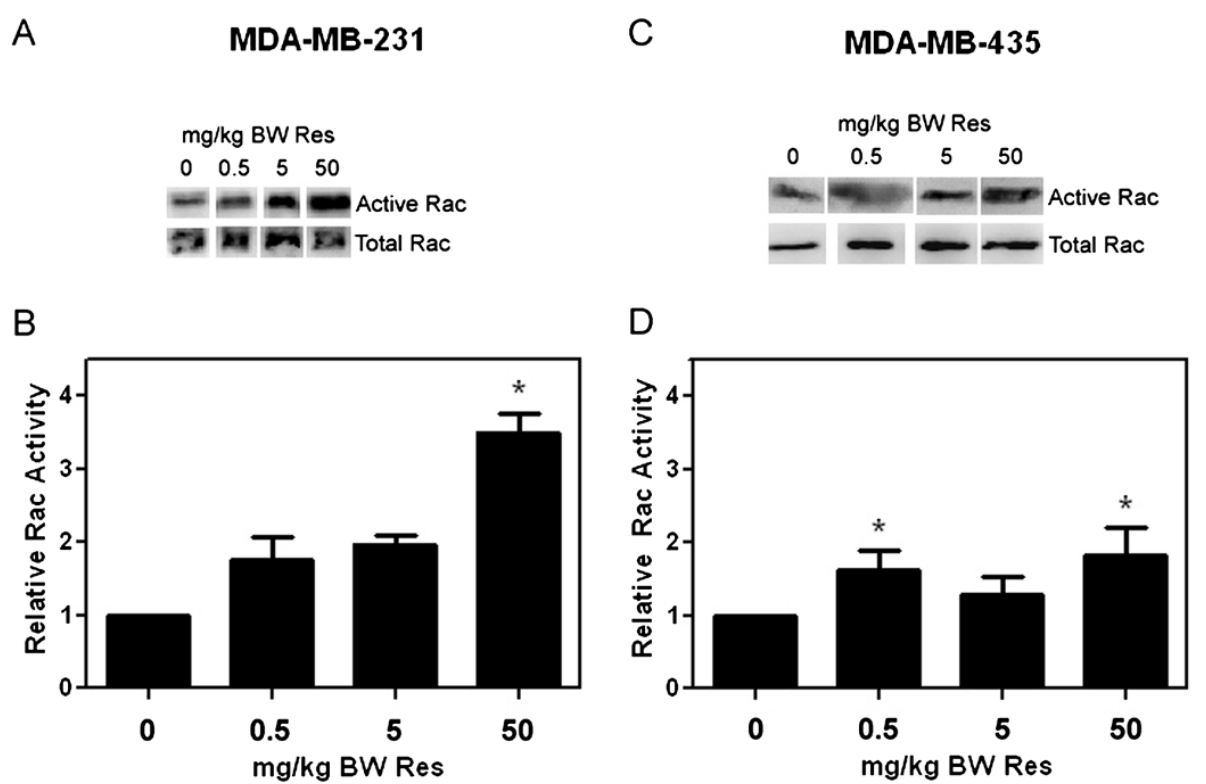

Figure 3 Rac activity in resveratrol treated tumors. GFP-MDA-MB-231 or GFP-MDA-MB-435 mammary tumors from mice that received vehicle or $0.5,5$, or $50 \mathrm{mg} / \mathrm{kg}$ BW Res diets were lysed. PAK-PBD-GST beads were used to pull down active (GTP-bound) Rac from tumor lysates. Active and total Rac levels were detected by western blot analysis with anti-Rac antibody. Rac activity (active Rac/total Rac) relative to vehicle control as quantified from densitometric scans of western blot analyses is presented for the study performed with (A and B), MDA-MB-231 and (C-D), MDAMB-435 cells. (A) and (C), Representative western blots for active (GTP-bound) Rac in the upper row and total tumoral Rac in the lower row for the MDA-MB-231 and the MDA-MB-435 studies, respectively. (B) and (D), Average relative Rac activity for the MDA-MB-231 and the MDA-MB-435 studies, respectively. Error bars represent SEMs from 2-4 tumors per group. Differences between means were determined using Student's t-Test. Asterisk denotes statistical significance at $p \leq 0.05$.

5 and $50 \mathrm{mg} / \mathrm{kg}$ BW resveratrol (Figure $1 \mathrm{~A}, \mathrm{~B}$, and C). Similarly, the growth of mammary fat pad tumors established in nude mice from the more aggressive and highly metastatic breast cancer cell line MDA-MB-435 was increased by resveratrol at all the concentrations tested. In the MDA-MB-435 study, the concentration that showed the greater effect was $0.5 \mathrm{mg} / \mathrm{kg}$ BW resveratrol with a $\sim 3$-fold increase, whereas 5 and $50 \mathrm{mg} / \mathrm{kg} \mathrm{BW}$ resveratrol increased tumor growth by $\sim 2.5$-fold (Figure 1D, E, and F). This increase in tumor growth presented with a $p>0.05$ compared to controls. This high probability may be due to the heterogeneity of individual tumor growth, because when we re-group into growing and poorly growing groups as cited in [42], there was a significant difference of 0.02 in the $5 \mathrm{mg} / \mathrm{kg}$ BW resveratrol group compared to vehicle controls. Moreover, administration of resveratrol at all concentrations tested did not result in significant differences in mouse weight among the resveratrol treated groups compared to vehicle controls (Additional file 1: Figure S1).

Even though our previous study, where we administered $5 \mathrm{mg} / \mathrm{kg}$ BW resveratrol in combination with equal amounts of quercetin and catechin, demonstrated reduced tumor growth and metastasis [26,34], in this study we found resveratrol alone induced a marked trend of increase in tumor growth, especially by the lower concentrations; thus, indicating a deleterious effect of resveratrol on breast cancer progression. This may suggest an alternate mechanism where resveratrol alone acts to promote tumor growth.

Our results highlight the importance of attaining a better understanding of the effects of resveratrol at low concentrations. Also noteworthy is the fact that $50 \mathrm{mg} / \mathrm{kg}$ BW resveratrol, which in other studies has been shown to reduce tumor growth in ovarian cancer and hepatocarcinoma $[38,40]$, induced tumor growth in both (MDAMB-231 and MDA-MB-435) breast cancer cells. This suggests that resveratrol effects are not only concentration dependent but can also vary depending on the cell and cancer type.

To investigate the role of resveratrol on breast cancer metastasis, lungs, livers, kidneys, and bones from mice bearing MDA-MB-231 and MDA-MB-435 mammary tumors were harvested at necropsy for identification of fluorescent metastatic foci. Mice from the MDA-MB-231 study only presented with lung metastasis; whereas, mice from the MDA-MB-435 study presented with metastasis to all harvested organs due to the highly metastatic potential of this cell line. Resveratrol dramatically induced lung metastasis in both studies. In mice bearing MDA-MB-231 derived mammary tumors, resveratrol at $0.5, \mathrm{mg} / \mathrm{kg} \mathrm{BW}$ caused a 30 -fold increase, and at 5 and $50 \mathrm{mg} / \mathrm{kg} \mathrm{BW}$, a significant 13- and 65-fold increase in lung metastasis 
A

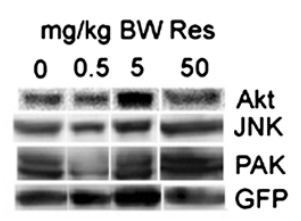

B

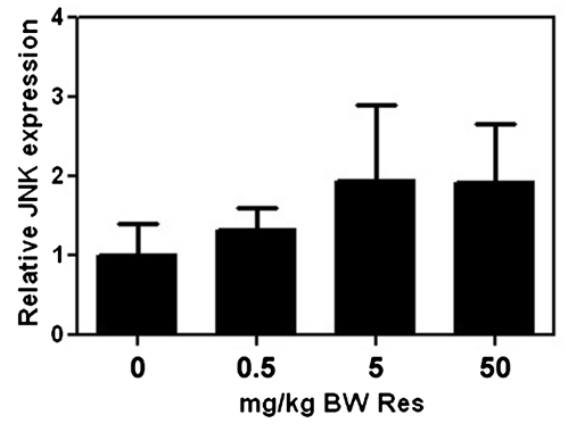

C

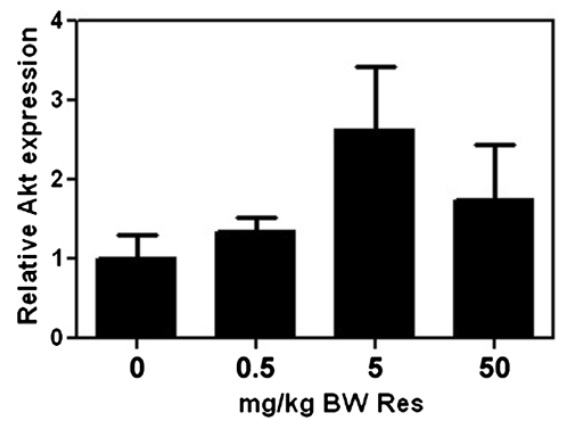

D

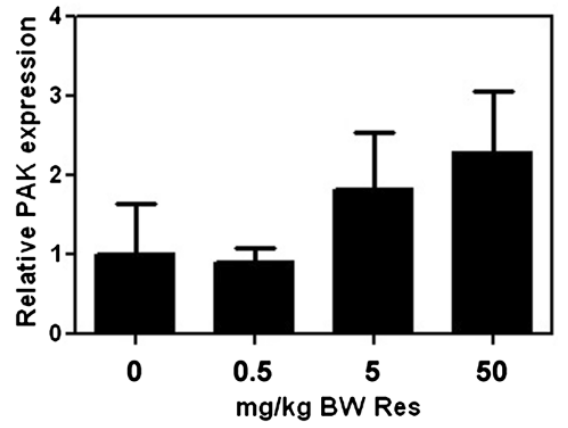

Figure 4 Effect of resveratrol on Akt, JNK, and PAK expression in mammary tumors. Total lysates of MDA-MB-231-derived mammary tumors from vehicle- and Res-treated mice were western blotted with anti-Akt, anti-JNK, or anti-PAK1 antibodies. Fold changes in protein expression were calculated from integrated density of positive bands and are presented relative to vehicle. Equal total protein content as well as confirmation of human cancer cells in the mouse tumor extracts was maintained by expressing the integrated density of each band as a function of the integrated density of the GFP band from the same tumor extract. (A), Representative western blots. (B), (C), and (D), Relative Akt, JNK, and PAK1 expression, respectively. Error bars represent SEMs from 2-4 tumors per group. Differences between means were determined using Student's t-Test.

after 108 days (Figure 2A). Likewise, in mice bearing MDA-MB-435 tumors, resveratrol at $0.5,5$, and $50 \mathrm{mg} / \mathrm{kg}$ BW caused an 11-, 36-, and 9-fold increase in lung metastasis, respectively, after only 44 days (Figure $2 \mathrm{~B}$ ). In the case of kidneys and bone, mice from the vehicle group did not present with metastasis while resveratrol-treated mice developed metastases (Table 1). Metastasis to the kidneys was observed in $14 \%, 10 \%$, and $22 \%$ of mice in $0.5,5$, and $50 \mathrm{mg} / \mathrm{kg}$ BW resveratrol treatment groups, respectively; whereas, metastasis to the bone was observed in $20 \%$ and $22 \%$ of mice treated with 5 and $50 \mathrm{mg} / \mathrm{kg} \mathrm{BW}$ resveratrol, respectively. Finally, liver metastases were identified in a similar percentage of mice from each treatment group, including vehicle control. However, the average integrated density was 10 - and 2.9- fold higher in 0.5 and $5 \mathrm{mg} / \mathrm{kg}$ BW resveratrol treated mice (Table 1), suggesting a higher number and/or larger metastatic foci in mice treated with low concentrations of resveratrol when compared to vehicle treated mice. Overall, the percentage of mice presented with metastasis was low for statistical comparisons due to the short duration of the study which, because of high tumor burden, had to be terminated after only 44 days.
Regardless, our findings strongly suggest that resveratrol at low concentrations can promote mammary tumor growth and metastasis.

Since resveratrol-mediated regulation of breast cancer cell migration/invasion was previously shown by us to be dependent on signaling from the small GTPase Rac [36], we investigated Rac activation status in MDA-MB-231 and MDA-MB-435-derived mammary tumors. In MDAMB-231 mammary tumors, resveratrol at $50 \mathrm{mg} / \mathrm{kg} \mathrm{BW}$ significantly increased Rac activity by 3.5 -fold; whereas 0.5 and $5 \mathrm{mg} / \mathrm{kg} \mathrm{BW}$ caused a non-significant increase in Rac activity of 1.75 and 2-fold, respectively (Figure 3A). On the other hand, in MDA-MB-435-derived tumors, resveratrol at 0.5 and $50 \mathrm{mg} / \mathrm{kg} \mathrm{BW}$ significantly increased Rac activity by 1.6- and 1.8-fold, respectively (Figure 3B). Our results suggest that resveratrol-mediated induction of metastasis might be, at least partly due to increased Rac activity.

We also investigated the effects of resveratrol in the tumoral expression of Akt and the MAPK jun kinase (JNK), key proteins in survival signaling and cell proliferation that are commonly disregulated in human 


\section{A}

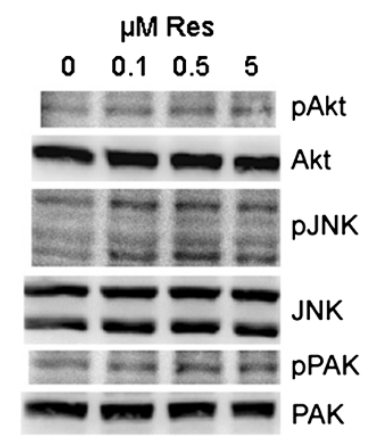

$\mathrm{B}$

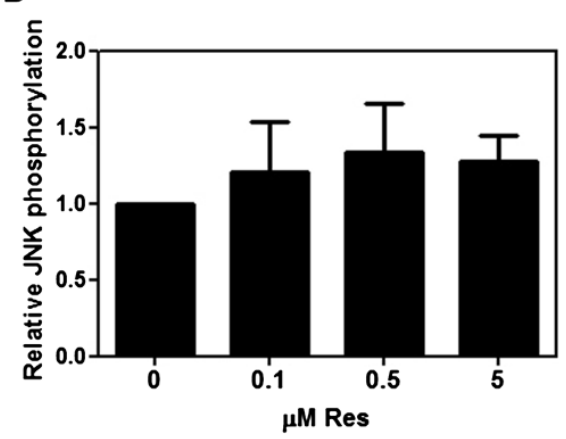

$\mathrm{C}$

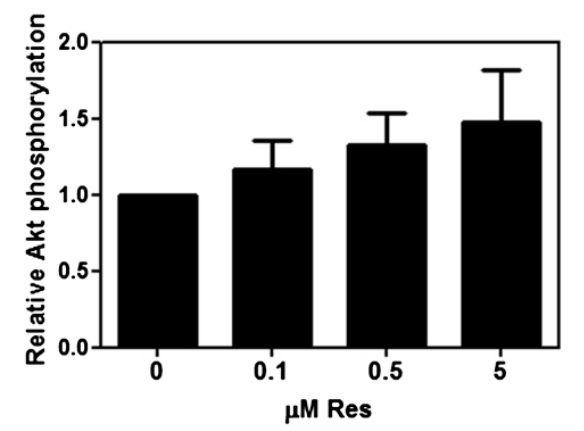

D

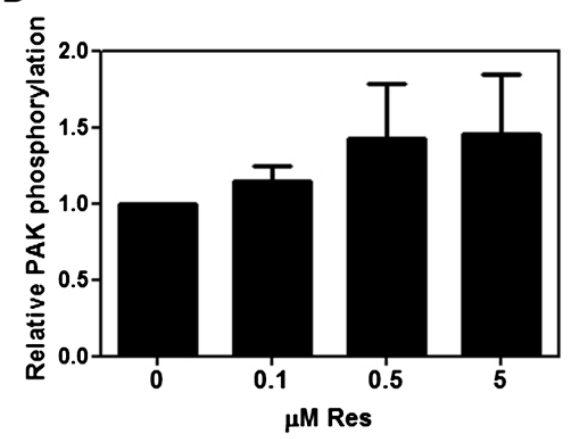

Figure 5 Effect of resveratrol on Akt, JNK, and PAK1 activity in MDA-MB-231 breast cancer cells. Confluent MDA-MB-231 cells were serum starved for $24 \mathrm{~h}$, treated with Veh, 0.1, 0.5, or $5 \mu \mathrm{M}$ resveratrol (Res) for 15 min, lysed immediately, and western blotted for active and total proteins: phospho-Akt ${ }^{\text {Thr308 }}$ and Akt, phospho-JNK ${ }^{\text {Thr183/Tyr185 }}$ and JNK, or phospho-PAK1 Thr431/Thr402 and PAK1. Fold changes in protein activity were calculated from integrated density of positive bands and are presented relative to vehicle. (A), Representative western blots. (B), (C), and (D), Relative Akt, JNK, and PAK1 activity (phosphorylated protein/total protein), respectively ( $\mathrm{n}=2-3$ ). Error bars represent SEMs. Differences between means were determined using Student's t-Test.

cancers and have been shown to be regulated by resveratrol $[4,6 ; 10-12 ; 38-40]$. In addition, we studied the expression of the Rac downstream effector $\mathrm{p}-21$ activated kinase 1 (PAK1), implicated in the regulation of cell motility/migration and therefore, metastasis. PAKs are also relevant for the control of other cellular processes important for tumorigenesis such as survival, proliferation and gene transcription [41], via regulation of Akt and MAPKs pathways [42]. We analyzed Akt, JNK, and PAK1 protein expression in MDA-MB-231 derived mammary tumors by western blot. Our results show a trend in upregulation (1.75-2.5-fold) of these kinases in tumors from mice that received resveratrol at 5 and $50 \mathrm{mg} / \mathrm{kg}$ BW; thus, implicating the PI3-K/Akt, MAPK, and Rac/ PAK pathways in potential effects of resveratrol on mammary tumor growth and metastasis (Figure 4).

To test whether the increased expression of Akt, JNK, and PAK1 resulted in parallel increases in their activity, we tested the effect of low concentrations of resveratrol on the active phosphorylated levels of these signaling proteins. Quiescent MDA-MB-231 or MDA-MB-435 cells were treated with $0.1,0.5$, and $5 \mu \mathrm{M}$ resveratrol for $15 \mathrm{~min}$ and the lysates western blotted using total or phsopho-specific antibodies to Akt, JNK, and PAK1. In parallel with increased expression of total proteins from the MDA-MB-231 mammary tumors in mice treated with resveratrol (Figure 4), we observed increased phosphorylation of Akt, JNK, and PAK1 by resveratrol at all concentrations tested in MDA-MB-231 cells (Figure 5), but not in MDA-MB-435 cells (data not shown). Therefore, the upregulated expression and activities of the Rac effectors PAK, JNK, and Akt, are expected to contribute to the observed increase in tumor growth and metastasis in response to resveratrol.

Even though resveratrol at high concentrations inhibits Akt and MAPK activities, at low concentrations, resveratrol has been previously shown to promote proliferation in human cancer cells and to induce Akt and MAPKs, among other tumorigenic signaling proteins [43]. Resveratrol modulates a plethora of signaling pathways important for tumor progression; and therefore, it would be too simplistic to limit resveratrol's cancer promoting (or inhibiting) properties to a few molecules or a single signaling pathway $[44,45]$. Thus, it is more reasonable to think that the collective activity of resveratrol, rather than a single effect, is responsible for our results. In addition to 
modulation of steroid receptor signaling and crosstalk with growth factor receptors that can affect both signaling and transcriptional activities [46], resveratrol was recently shown to exhibit dose dependent regulation of Wnt signaling and the expression of Wnt target genes [47]. Since resveratrol affected mammary tumor growth in both $\mathrm{ER} \alpha(-), \mathrm{ER} \beta(+)$ MDA-MB-231 and ER(-) MDA-MB-435 cancer cells, ER independent mechanisms may be responsible for our intriguing findings. Such mechanisms can include: 1) the regulation of ER related receptors that are active in ER(-) breast cancers [48] and are regulated by phytoestrogens [49], or 2) the collective regulation of multiple signaling and transcriptional pathways.

\section{Conclusions}

Resveratrol is widely known for its anti-cancer properties. However, our study implicates low concentrations of resveratrol on promotion of breast cancer progression and metastasis and implicates tumorigenic and metastatic signaling pathways in resveratrol's tumor promoting effects. The present study clearly demonstrates that resveratrol, depending on the dose, can induce growth and metastasis of breast cancers. Therefore, prior to dietary recommendations of resveratrol or clinical trials as an anti-cancer therapeutic, it is critical to have a better understanding of resveratrol effects at physiological concentrations. Moreover, our findings serve to caution patients or physicians in the use of this grape polyphenol as an alternative and/or complementary medicine for breast cancer treatment.

\section{Additional file}

Additional file 1: Figure S1. Effect of resveratrol on mice weight. GFP-tagged MDA-MB-231 or MDA-MB-435 cells $\left(1 \times 10^{6}\right)$ in Matrigel: DMEM (1:1) were inoculated at the mammary fat pad of female SCID (MDA-MB-231) or athymic nude mice (MDA-MB-435). One week following injection, mice were fed vehicle (Veh) or $0.5,5$, or $50 \mathrm{mg} / \mathrm{kg} \mathrm{BW}$ resveratrol (Res) 5 times a week by oral gavage. Mice weights were recorded every two weeks to monitor toxicity and are presented for (A) the MDA-MB-231 study and (B) the MDA-MB-435 study, as a function of days. Differences between means were determined using Student's t-Test.

Competing interests

The authors declare that they have no competing interests.

\section{Authors' contributions}

LCP contributed to the experimental design, conducted all experiments and analyzed and interpreted data. LCP also wrote the manuscript. LAC was involved with critical revision of the manuscript. SD contributed to the experimental design, directed the research, assisted with animal protocols, and data analysis and interpretation. She also contributed to writing and critical revision of the manuscript. All authors read and approved the final manuscript.

\section{Acknowledgements}

This study is sponsored by the Department of Defense/Breast Cancer Research Program awards W81XWH-07-1-0330 to SD and W81XWH-08-01-0258 to LCP, NIH/NIGMS SC3GM084824 to SD,
NIH/NCRR grants 2G12RR003035 to UCC and G12-RR03051 to UPR MSC. We thank Dr. Aldo Pérez for his excellent technical assistance with animal protocols, Malika S Flanagan for assistance with fluorescence image analysis of metastatic foci, and Jeyshka M. Reyes for assistance with western blotting. We also acknowledge the services of the Animal Resources Center, Universidad Central del Caribe, Bayamón, Puerto Rico.

\section{Author details}

'Department of Biochemistry, School of Medicine, University of Puerto Rico, Medical Sciences Campus, PO Box 365067, San Juan, Puerto Rico.

${ }^{2}$ Department of Pathology and Laboratory Medicine, Universidad Central de Caribe School of Medicine, Bayamon, Puerto Rico. ${ }^{3}$ Department of Anatomy and Cell Biology, Universidad Central del Caribe School of Medicine, Bayamon, Puerto Rico.

Received: 20 July 2012 Accepted: 2 January 2013

Published: 8 January 2013

\section{References}

1. Gomez-Serranillos MP, Martin S, Ortega T, Palomino OM, Prodanov M, Vacas $V$, et al: Study of red wine neuroprotection on astrocytes. Plant Foods Hum Nutr 2009, 64:238-243.

2. Xia EQ, Deng GF, Guo YJ, Li HB: Biological activities of polyphenols from grapes. Int J Mol Sci 2010, 11:622-646.

3. Ndiaye M, Kumar R, Ahmad N: Resveratrol in cancer management: where are we and where we go from here? Ann N Y Acad Sci 2011, 1215:144-149.

4. Shukla Y, Singh R: Resveratrol and cellular mechanisms of cancer prevention. Ann N Y Acad Sci 2011, 1215:1-8.

5. Fulda S, Debatin KM: Resveratrol modulation of signal transduction in apoptosis and cell survival: a mini-review. Cancer Detect Prev 2006, 30:217-223.

6. Bhat KP, Lantvit D, Christov K, Mehta RG, Moon RC, Pezzuto JM: Estrogenic and antiestrogenic properties of resveratrol in mammary tumor models. Cancer Res JID - 2984705R 2001, 61:7456-7463.

7. Bowers JL, Tyulmenkov W, Jernigan SC, Klinge CM: Resveratrol acts as a mixed agonist/antagonist for estrogen receptors alpha and beta. Endocrinology 2000, 141:3657-3667.

8. Levenson AS, Gehm BD, Pearce ST, Horiguchi J, Simons LA, Ward JE III, et al: Resveratrol acts as an estrogen receptor (ER) agonist in breast cancer cells stably transfected with ER alpha. Int J Cancer 2003, 104:587-596.

9. Filomeni G, Graziani I, Rotilio G, Ciriolo MR: Trans-Resveratrol induces apoptosis in human breast cancer cells MCF-7 by the activation of MAP kinases pathways. Genes Nutr 2007, 2:295-305.

10. Nguyen TH, Mustafa FB, Pervaiz S, Ng FS, Lim LH: ERK1/2 activation is required for resveratrol-induced apoptosis in MDA-MB-231 cells. Int J Oncol 2008, 33:81-92.

11. Alkhalaf M: Resveratrol-induced growth inhibition in MDA-MB-231 breast cancer cells is associated with mitogen-activated protein kinase signaling and protein translation. Eur J Cancer Prev 2007, 16:334-341.

12. De AF, Giordano F, Vivacqua A, Pellegrino M, Panno ML, Tramontano D, et al: Resveratrol, through NF-Y/p53/Sin3/HDAC1 complex phosphorylation, inhibits estrogen receptor \{alpha\} gene expression via p38MAPK/CK2 signaling in human breast cancer cells. FASEB J 2011, 25:3695-707.

13. Araujo JR, Goncalves P, Martel F: Chemopreventive effect of dietary polyphenols in colorectal cancer cell lines. Nutr Res 2011, 31:77-87.

14. Szekeres T, Saiko P, Fritzer-Szekeres M, Djavan B, Jager W: Chemopreventive effects of resveratrol and resveratrol derivatives. Ann N Y Acad Sci 2011, 1215:89-95

15. Gupta SC, Kannappan R, Reuter S, Kim JH, Aggarwal BB: Chemosensitization of tumors by resveratrol. Ann N Y Acad Sci 2011 , 1215:150-160

16. Brownson DM, Azios NG, Fuqua BK, Dharmawardhane SF, Mabry TJ: Flavonoid effects relevant to cancer. J Nutr JID - 04042432002 132:3482S-34895.

17. Azios NG, Dharmawardhane SF: Resveratrol and estradiol exert disparate effects on cell migration, cell surface actin structures, and focal adhesion assembly in MDA-MB-231 human breast cancer cells. Neoplasia 2005, 7:128-140. 
18. Johnson JJ, Nihal M, Siddiqui IA, Scarlett CO, Bailey HH, Mukhtar $\mathrm{H}$, et al: Enhancing the bioavailability of resveratrol by combining it with piperine. Mol Nutr Food Res 2011, 55:1169-1176.

19. Santos AC, Veiga F, Ribeiro AJ: New delivery systems to improve the bioavailability of resveratrol. Expert Opin Drug Deliv 2011, 8:973-990.

20. Garvin S, Ollinger K, Dabrosin C: Resveratrol induces apoptosis and inhibits angiogenesis in human breast cancer xenografts in vivo. Cancer Lett 2006, 231:113-122.

21. Osmond GW, Augustine CK, Zipfel PA, Padussis J, Tyler DS: Enhancing melanoma treatment with resveratrol. J Surg Res 2012, 172:109-115.

22. Niles RM, Cook CP, Meadows GG, Fu YM, McLaughlin JL, Rankin GO: Resveratrol is rapidly metabolized in athymic (nu/nu) mice and does not inhibit human melanoma xenograft tumor growth. J Nutr 2006, 136:2542-2546.

23. Fukui M, Yamabe N, Zhu BT: Resveratrol attenuates the anticancer efficacy of paclitaxel in human breast cancer cells in vitro and in vivo. Eur J Cancer 2010, 46:1882-1891.

24. Patel KR, Scott E, Brown VA, Gescher AJ, Steward WP, Brown K: Clinical trials of resveratrol. Ann N Y Acad Sci 2011, 1215:161-169.

25. Smoliga JM, Baur JA, Hausenblas HA: Resveratrol and health-a comprehensive review of human clinical trials. Mol Nutr Food Res 2011 55:1129-1141.

26. Castillo-Pichardo L, Martinez-Montemayor MM, Martinez JE, Wall KM, Cubano LA, Dharmawardhane S: Inhibition of mammary tumor growth and metastases to bone and liver by dietary grape polyphenols. Clin Exp Metastasis 2009, 26:505-516.

27. Lacroix M: MDA-MB-435 cells are from melanoma, not from breast cancer. Cancer Chemother Pharmacol 2009, 63:567.

28. Sellappan S, Grijalva R, Zhou X, Yang W, Eli MB, Mills GB, et al: Lineage infidelity of MDA-MB-435 cells: expression of melanocyte proteins in a breast cancer cell line. Cancer Res 2004, 64:3479-3485.

29. Chambers AF: MDA-MB-435 and M14 cell lines: identical but not M14 melanoma? Cancer Res 2009, 69:5292-5293.

30. Yang M, Baranov E, Jiang P, Sun FX, Li XM, Li L, et al: Whole-body optical imaging of green fluorescent protein-expressing tumors and metastases. Proc Natl Acad Sci USA 2000, 97:1206-1211.

31. Hoffman RM, Yang M: Whole-body imaging with fluorescent proteins. Nat Protoc 2006, 1:1429-1438.

32. Hoffman RM: The multiple uses of fluorescent proteins to visualize cancer in vivo. Nat Rev Cancer 2005, 5:796-806.

33. Carlson AL, Hoffmeyer MR, Wall KM, Baugher PJ, Richards-Kortum R, Dharmawardhane SF: In situ analysis of breast cancer progression in murine models using a macroscopic fluorescence imaging system. Lasers Surg Med 2006, 38:928-938.

34. Schlachterman A, Valle F, Wall KM, Azios NG, Castillo L, Morell L, et al: Combined resveratrol, quercetin, and catechin treatment reduces breast tumor growth in a nude mouse model. Trans/ Oncol 2008, 1:19-27.

35. Martinez-Montemayor MM, Otero-Franqui E, Martinez J, De LM-P, Cubano LA, Dharmawardhane S: Individual and combined soy isoflavones exert differential effects on metastatic cancer progression. Clin Exp Metastasis 2010, 27:465-480.

36. Azios NG, Krishnamoorthy L, Harris M, Cubano LA, Cammer M Dharmawardhane SF: Estrogen and resveratrol regulate Rac and Cdc42 signaling to the actin cytoskeleton of metastatic breast cancer cells. Neoplasia 2007, 9:147-158.

37. Le CC, Carlier MF: Regulation of actin assembly associated with protrusion and adhesion in cell migration. Physiol Rev 2008, 88:489-513.

38. Lee MH, Choi BY, Kundu JK, Shin YK, Na HK, Surh YJ: Resveratrol suppresses growth of human ovarian cancer cells in culture and in a murine xenograft model: eukaryotic elongation factor $1 \mathrm{~A} 2$ as a potential target. Cancer Res 2009, 69:7449-7458.

39. Chen J, Dong XS, Guo XG: Inhibitory effect of resveratrol on the growth of human colon cancer Is174t cells and its subcutaneously transplanted tumor in nude mice and the mechanism of action. Zhonghua Zhong Liu Za Zhi 2009, 31:15-19.

40. Bishayee A, Dhir N: Resveratrol-mediated chemoprevention of diethylnitrosamine-initiated hepatocarcinogenesis: inhibition of cell proliferation and induction of apoptosis. Chem Biol Interact 2009, 179:131-144.
41. Perez-Vizcaino F, Duarte J, Santos-Buelga C: The flavonoid paradox: conjugation and deconjugation as key steps for the biological activity of flavonoids. J Sci Food Agric 2012, 92:1822-1825.

42. Laajala TD, Corander J, Saarinen NM, Makela K, Savolainen S, Suominen MI, et al: Improved statistical modeling of tumor growth and treatment effect in preclinical animal studies with highly heterogeneous responses in vivo. Clin Cancer Res 2012, 18:4385-4396.

43. Mukherjee S, Dudley Jl, Das DK: Dose-dependency of resveratrol in providing health benefits. Dose-Response 2010, 8:478-500.

44. Whitlock NC, Baek SJ: The anticancer effects of resveratrol: modulation of transcription factors. Nutr Cancer 2012, 64:493-502.

45. Pezzuto JM: The phenomenon of resveratrol: redefining the virtues of promiscuity. Ann N Y Acad Sci 2011, 1215:123-130.

46. Wang $Y$, Romigh $T$, He $X$, Orloff MS, Silverman RH, Heston WD, et al: Resveratrol regulates the PTEN/AKT pathway through androgen receptor-dependent and -independent mechanisms in prostate cancer cell lines. Hum Mol Genet 2010, 19:4319-4329.

47. Chen HJ, Hsu LS, Shia YT, Lin MW, Lin CM: The beta-catenin/TCF complex as a novel target of resveratrol in the $\mathrm{Wnt} /$ beta-catenin signaling pathway. Biochem Pharmacol 2012, 84:1143-1153.

48. Chang CY, McDonnell DP: Molecular pathways: the metabolic regulator estrogen-related receptor alpha as a therapeutic target in cancer. Clin Cancer Res 2012, 18:6089-6095.

49. Ariazi EA, Jordan VC: Estrogen-related receptors as emerging targets in cancer and metabolic disorders. Curr Top Med Chem 2006, 6:203-215.

doi:10.1186/1472-6882-13-6

Cite this article as: Castillo-Pichardo et al.: Dietary grape polyphenol resveratrol increases mammary tumor growth and metastasis in immunocompromised mice. BMC Complementary and Alternative Medicine 2013 13:6.

\section{Submit your next manuscript to BioMed Central and take full advantage of:}

- Convenient online submission

- Thorough peer review

- No space constraints or color figure charges

- Immediate publication on acceptance

- Inclusion in PubMed, CAS, Scopus and Google Scholar

- Research which is freely available for redistribution 